\title{
Subcellular distribution of HP1 proteins is altered in ICF syndrome
}

\author{
Judith J Luciani ${ }^{1}$, Danielle Depetris ${ }^{1}$, Chantal Missirian ${ }^{2}$, Cécile Mignon-Ravix ${ }^{1}$, Catherine \\ Metzler-Guillemain $^{1}$, André Megarbane ${ }^{3}$, Anne Moncla ${ }^{2}$ and Marie-Geneviève Mattei ${ }^{\star 1}$
}

\author{
${ }^{1}$ Inserm U 491, Faculté de Médecine, Marseille, France; ${ }^{2}$ Département de Génétique, Hôpital d'enfants de la Timone, \\ Marseille, France; ${ }^{3}$ Departement de Génétique Médicale, Université Saint Joseph, Beyrouth, Liban
}

The Immunodeficiency, Centromeric instability, and Facial (ICF) syndrome is a rare autosomal recessive disorder that results from mutations in the DNMT3B gene, encoding a DNA-methyltransferase that acts on GC-rich satellite DNAs. This syndrome is characterized by immunodeficiency, facial dysmorphy, mental retardation of variable severity and chromosomal abnormalities that essentially involve juxtacentromeric heterochromatin of chromosomes 1 and 16. These abnormalities demonstrate that hypomethylation of satellite DNA can induce alterations in the structure of heterochromatin. In order to investigate the effect of DNA hypomethylation on heterochromatin organization, we analyzed the in vivo distribution of HP1 proteins, essential components of heterochromatin, in three ICF patients. We observed that, in a large proportion of ICF G2 nuclei, all HP1 isoforms show an aberrant signal concentrated into a prominent bright focus that co-localizes with the undercondensed 1qh or 16qh heterochromatin. We found that SP100, SUMO-1 and other proteins from the promyelocytic leukemia nuclear bodies (NBs) form a large body that co-localizes with the HP1 signal. This is the first description of altered nuclear distribution of HP1 proteins in the constitutional ICF syndrome. Our results show that satellite DNA hypomethylation does not prevent HP1 proteins from associating with heterochromatin. They suggest that, at G2 phase, HP1 proteins are involved in the heterochromatin condensation and may therefore remain concentrated at these sites until the condensation is complete. They also indicate that proteins from the NB could play a role in this process. Finally, satellite DNA length polymorphism could affect the efficiency of heterochromatin condensation and thus contribute to the variability of the ICF phenotype.

European Journal of Human Genetics (2005) 13, 41-51. doi:10.1038/sj.ejhg.5201293

Published online 6 October 2004

Keywords: ICF syndrome; HP1 proteins; heterochromatin; chromosome condensation; PML nuclear body

\section{Introduction}

The Immunodeficiency, Centromeric instability, and Facial dysmorphy (ICF) syndrome (MIM 242860) is a rare autosomal recessive disorder. Patients with ICF syndrome suffer from recurrent respiratory and/or gastrointestinal infections and have facial anomalies, as well as mental

*Correspondence: Dr M-G Mattei, Genetique Medicale et Developpement, INSERM U491, Faculté de Médecine, 27 Boulevard Jean Moulin, F-13385 Marseille, France. Tel: + 3304912571 73; Fax: + 3304918043 19; E-mail: genevieve.mattei@medecine.univ-mrs.fr

Received 26 May 2004; revised 28 July 2004; accepted 18 August 2004 retardation of variable severity. Moreover, juxtacentromeric abnormalities involving chromosomes 1 and 16, and to a lesser extent chromosome 9, are the diagnostic hallmarks of the syndrome. ${ }^{1}$ The majority of ICF cases display mutations in the DNA-methyltransferase $3 \mathrm{~B}$ (DNMT3B) gene. ${ }^{2-4}$ The specific role of DNMT3B methyltransferase appears to be the de novo methylation of the GCrich classical satellite DNAs. In ICF patients, there is therefore constitutive hypomethylation of satellite 2 DNA, mostly located at the juxtacentromeric heterochromatin of chromosomes 1 (1qh) and 16 (16qh), and of satellite 3 DNA which is mostly located at the $9 \mathrm{qh}$ heterochromatin. ${ }^{5,6}$ The 
cytogenetic abnormalities detectable in mitogen-stimulated lymphocytes, as well as in lymphoblastoid cell lines, are specifically targeted to these heterochromatic regions and include marked elongation of juxtacentromeric heterochromatin, multibranched chromosome configurations, centromeric breakages, whole arm chromosome deletion or duplication and micronuclei containing acentric fragments. Such observations have led to the suggestion that hypomethylation of satellite DNAs induces alterations in the structure, condensation and stability of the heterochromatin in these regions.

In addition to methylated DNA, the heterochromatin protein 1 (HP1) is a major component of heterochromatin and plays a key role in its formation and maintenance in Drosophila $^{7,8}$ and in various mammalian species. ${ }^{9-11}$ In humans, three HP1 protein isoforms, HP1 $\alpha, \mathrm{HP} 1 \beta$ and $\mathrm{HP} 1 \gamma$, have been isolated, which all co-localize with constitutive heterochromatin, suggesting that they are involved in its organization. ${ }^{12,13}$ This involvement is now known to occur through their interaction with SUVAR39H1, a histone methyltransferase which methylates histone $\mathrm{H} 3$ at lysine 9 (H3-K9). HP1 binds to methylated H3-K9 through its chromodomain and in turn recruits SUVAR39H1, which further propagates methylation along the chromatin. ${ }^{12,14,15}$

To gain insight into the mechanisms by which DNA hypomethylation may influence the organization of the heterochromatin, we therefore analyzed the in vivo distribution of HP1 $\alpha, \mathrm{HP} 1 \beta$ and HP $1 \gamma$ proteins in the cell lines of three ICF patients. We showed by immuno-FISH that a large proportion of ICF nuclei presented an aberrant HP1 signal concentrated into a prominent bright focus, colocalizing with $1 \mathrm{qh}$ or $16 \mathrm{qh}$ heterochromatin. By synchronizing the cells, we demonstrated that the nuclei with a bright HP1 focus were specific to the G2 phase of the cell cycle. Moreover, using a number of antibodies directed against proteins that interact directly, or indirectly, with HP1, we found that the prominent HP1 focus always colocalizes with SP100, SUMO-1 and other proteins of the ProMyelocytic Leukemia (PML) nuclear bodies (NBs that are disrupted in $\mathrm{PML}^{16}$ ).

We discuss our results with respect to the molecular and cytogenetic characteristics of ICF syndrome and the major components involved in heterochromatin organization.

\section{Material and methods Patients}

Three ICF patients were studied, ICF1, ICF2 and ICF3.

Patient ICF1 is a female patient first referred to our hospital at the age of 9 months for dysmorphy and severe infections related to agammaglobulinemia. Cytogenetic studies, performed on PHA-stimulated lymphocytes, showed the characteristic abnormalities of the ICF syndrome, involving the heterochromatin regions of chromo- somes 1 and 16. This child is now 3 years old and presents delayed psychomotor development and growth retardation ( -2 SD). No mutation has been found in the coding region of the DNMT3B gene (Viegas-Peguignot, unpublished data, 2004). Fibroblasts isolated from skin biopsy were also obtained for this patient.

Patient ICF2 is a female patient aged 4 years, diagnosed on the association of discrete facial dysmorphism and agammaglobulinemia leading to severe infections. She presents normal psychomotor development and no growth retardation. Cytogenetic studies, performed on PHAstimulated lymphocytes, supported the diagnosis of ICF syndrome. In this patient, a single mutation was identified in the DNMT3B gene (Viegas-Peguignot, unpublished data, 2004).

Patient ICF3 is a female patient described elsewhere, ${ }^{17}$ whose lymphoblastoid cell line is available from the Coriell Cell Repositories (GM 08714, Camden, NJ). Chromosome analysis performed on lymphoblastoid cell line showed typical ICF aberrations involving chromosomes 1, 16 and rarely chromosome 9 . We also observed the addition of a chromosome 1 short arm in about $12 \%$ of the cells. This patient was a compound heterozygote as two different mutations were found in the DNMT3B gene, which were located in the catalytic domain of the DNMT3B methyltransferase. ${ }^{2}$

Controls C1, C2 and C3 are one male and two female individuals of less than 18 years of age, who are in normal health.

\section{Cytogenetic preparations}

We used both PHA-stimulated lymphocytes and freshly EBV-transformed lymphoblastoid cell lines from patients ICF1, ICF2 and control subjects. We only used the lymphoblastoid cell line from the ICF3 patient. Cell cultures were treated with our usual high-resolution banding method: 5-fluorodeoxyuridine/uridine (FdUR/Ur) added for $17 \mathrm{~h}$ at final concentrations of 1.8 and $4 \mu \mathrm{M}$, respectively, and release of the block by addition of thymidine for $7 \mathrm{~h}$ (final concentration: $10 \mu \mathrm{M}$ ), with colchicin added for the last hour.

\section{Cell synchronization}

Both control and ICF lymphoblastoid cell lines were treated in the same way with a variety of protocols in order to synchronize them at different phases of the cell cycle.

A double aphidicolin block was used to synchronize the cells at late G1 phase (G1/S boundary) as described. ${ }^{18}$ Aphidicolin specifically inhibits nuclear DNA synthesis in eukaryotic cells by interacting with the replicative DNA polymerase alpha. For synchronization at S-phase, cells were exposed for $17 \mathrm{~h}$ to a 5-FdUR/Ur block at final concentrations of 1.8 and $4 \mu \mathrm{M}$, respectively. FdUR, at the concentration used, is a strong and very specific inhibitor 
of thymidylate synthetase ${ }^{19}$ and the cells accumulate in mid-S phase before being collected. To synchronize cells at G2 phase, the FdUR/Ur block was released by the addition of thymidine at a final concentration of $10 \mu \mathrm{M} .{ }^{19}$ Upon removal of the block, cells that are arrested at the mid-S phase recover immediately, and when collected $5 \mathrm{~h}$ later they are assumed to be in G2 phase. Finally, in order to collect a large majority of cells at metaphase (M phase), the FdUR/Ur block was released by addition of thymidine for $12 \mathrm{~h}$, with colchicin added for the last $8 \mathrm{~h}$, before the cells were collected.

For each cell synchronization step, about 200 nuclei were analyzed after immunocytochemistry with HP1antibodies, in all ICF patients and control subjects.

\section{Molecular cytogenetics}

The fluorescent in situ hybridization (FISH) method was used both to characterize cytogenetic abnormalities in the cells of ICF patients and to localize various satellite DNAs with respect to the HP1 protein signals. To exclude potential artifacts in the immuno-FISH procedure, experiments were performed in a sequential manner, as described previously. ${ }^{20}$ Probes were chosen that allowed the identification of chromosomes (Chr) and heterochromatin regions specifically rearranged in the ICF syndrome.

Chr 1 was identified with a DNA paint probe (Cat: 1066-1 B-02, Adgenix, Voisins le Bretonneux, France), a probe specific for centromeres of Chr 1, 5 and 19 (Cat: LPE 05, Amplitech, Compiègne, France) and a probe specific for the 1qh heterochromatin (Cat: PSAT 0001, Q-Biogene, Illkirch, France).

Chr 16 was identified with a DNA paint probe (Cat: P5203, Q-Biogene). No probe exists which specifically labels the 16qh heterochromatin, and we therefore used a probe that labels the adjacent centromeric region (Cat: PSAT 0016, Q-Biogene).

The 9qh heterochromatic region was unambiguously identified with a specific probe (Cat: CP5016, Q-Biogene).

\section{Immunocytochemistry}

Preparations of nuclei from lymphocytes and lymphoblastoid cells were collected by centrifugation and prepared as described previously. ${ }^{20}$ Fibroblasts were trypsinized, rinsed and resuspended in PBS ( $\mathrm{pH}$ 7.2), but were then treated as the other cell types. Cells were centrifugated $(300 \mathrm{t} / \mathrm{min}$ for $5 \mathrm{~min}$ ) onto polylysine-coated slides in cytospin 3 (Shandon, Cergy-Pontoise, France), then fixed at room temperature (RT) for $10 \mathrm{~min}$ in $2 \%(\mathrm{w} / \mathrm{v})$ paraformaldehyde/PBS (pH 7.2).

For indirect immunofluorescence experiments, each primary antibody was diluted and used under specific conditions, then detected as described previously. ${ }^{20}$ After immunocytochemistry, all preparations were fixed with $1 \%$ $(\mathrm{w} / \mathrm{v})$ paraformaldehyde in PBS for $5 \mathrm{~min}$, rinsed again before staining with DAPI $(100 \mathrm{ng} / \mathrm{ml})$ diluted in Vectashield (Vector Laboratories). All antibodies were tested in indivi- dual staining reactions for their specificity and performance. Controls without primary antibody were all negative.

\section{Antibodies (Abs)}

The three anti-HP1 mouse Abs, 2HP-1H5, 1MOD-1A9 and 2MOD-1G6, directed against HP1 $\alpha, \mathrm{HP} 1 \beta$ and HP1 $\gamma$, respectively, were kindly provided by $\mathrm{P}$ Chambon (IGBMC, Illkirch, France). We also used the branched anti$4 \times$ dimethyl-histone H3-Lys9 rabbit Ab, kindly provided by $\mathrm{T}$ Jenuwein (IMP, Vienna, Austria) and used as recommended by A Peters (IMP, Vienna, Austria). The anti-Ikaros rabbit $\mathrm{Ab}$ was a gift from A Fisher (MRC, London, UK); the mouse 7C2 $\mathrm{Ab}$, directed against phosphorylated CTD of RNA Polymerase II, was kindly provided by M Vigneron \& C Kedinger (ESBS, Strasbourg, France); the rabbit anti-histone $\mathrm{H} 3$ acetylated on both Lys 9 and Lys $18 \mathrm{Ab}$ was a kind gift from S Dent (DBMB, Houston, TX, USA); the rabbit anti-histone $\mathrm{H} 4$ acetylated on Lys5, Lys8, Lys12 and/or Lys16 Ab was kindly provided by C Vourc'h and C Jolly (IAB, La Tronche, France). The anti-SP100 is a rabbit Ab from Chemicon International, (Cat: AB1380). Several other Abs were purchased from Upstate Biotechnology: the mouse anti-SUVAR39H1 Ab (Cat: 05-615), the rabbit anti-dimethyl-histone H3 (Lys 9) Ab (Cat: 07-212), the rabbit anti-MeCP2 Ab (Cat: 07-013), the rabbit antiDAXX Ab (Cat: 07-411) and the mouse anti-HDAC1 $\mathrm{Ab}$ (Cat: 05-614). Other Abs were purchased from Santa Cruz Biotechnology: the mouse anti-PML (Cat: sc-966) Ab, the rabbit anti-SUMO-1 Ab (Cat: sc-9060), the rabbit anti-mi2 $\mathrm{Ab}$ (Cat: sc-11378), the goat Abs anti Dnmt3b (Cat: sc-10235) and anti-Dnmt3b (Cat: sc-10236), the rabbit anti-Dnmt3b Ab (Cat:sc-20704) and the rabbit anti-Dnmt1 Ab (Cat:sc-20701).

\section{Microscopic analysis}

Nuclei preparations were observed using an Axioplan-2 Zeiss fluorescent microscope (Carl ZEISS, Le Pecq, France) and the images captured with a CCD camera (Photometrics 'Sensys', Tucson, AZ, USA). Information was collected and merged using IPLab Spectrum software (Vysis, Voisins le Bretonneux, France). In order to more precisely analyze the co-localization of the HP1 focus with either satellite DNA or other proteins, several nuclei were photographed in $3 \mathrm{D}$, using an axioplan-2 imaging Zeiss microscope equipped with a LUDL platform, motorized in $x, y$ and $z$ axes (Adgenix, Voisins le Bretonneux, France). This equipment allowed us to capture the images in different nuclear sections.

\section{Results \\ Cytogenetic abnormalities are characteristic and distinguish between our ICF patients}

We performed FISH on cytogenetic preparations of ICF lymphoblastoid cell lines treated with the high-resolution 
method. All chromosomal abnormalities observed in these preparations were characteristic of the syndrome and were similar to those observed in PHA-stimulated lymphocytes (our data and Carpenter et $a l^{17}$ ): broken chromosomes, multi-branched configurations and chromosome elongation with heterochromatin length greater than the short arm of the corresponding chromosome.

Interestingly, not only the three cell lines were different with respect to the total number of chromosomal abnormalities, ICF1: 39\%, ICF2: 23\% and ICF3: 28\%, but they could also be distinguished by the degree of involvement of Chr 1 and 16 in these abnormalities. Indeed, in both the ICF1 and ICF3 cell lines, the 1qh heterochromatin regions were more frequently involved than the 16qh ones in breakages and multi-branched configurations, while Chr 9 was rarely involved (Figure 1a). In contrast, in the ICF2 cell line, Chr 1 and 16 were involved in cytogenetic abnormalities with a nearly equivalent frequency (Figure 1b).

On the same preparations, we analyzed the chromosomal content of micronuclei, structures that are also characteristic of ICF syndrome. Micronuclei are expected to contain acentric chromosome fragments, and thus reflect genetic loss from one or both daughter cells at mitosis. We observed a higher number of micronuclei in the ICF1 (12\%) and 1CF3 (10\%) cell lines than in the ICF2 (6\%) cell line. Moreover, we demonstrated that Chr 1 and 16 have an incidence of inclusion in micronuclei that
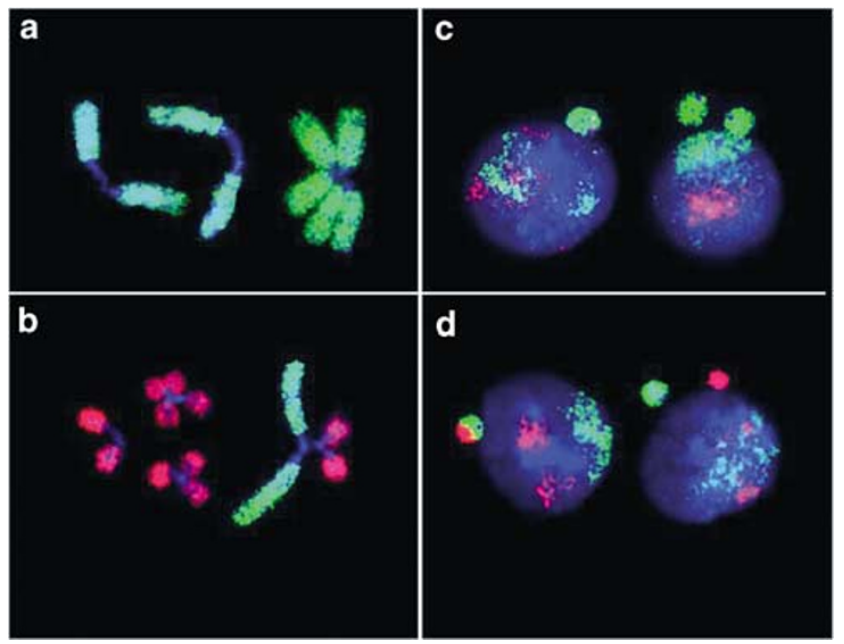

Figure 1 Chromosomal abnormalities in metaphasic and interphasic cells of ICF patients: dual-color FISH was performed with chromosome 1 (green) and chromosome 16 (red) paint probes. Chromosomes and nuclei are counterstained with DAPI (blue). (a) and (c) show chromosomal abnormalities and micronuclei involving specifically chromosome 1 , as frequently observed in the ICF1 and 1CF3 cell lines. (b) and (d) show chromosomal abnormalities and micronuclei involving both chromosomes 1 and 16, as frequently observed in the ICF2 cell line. reflects their participation in chromosomal abnormalities detected in metaphases. Therefore, the ICF1 and ICF3 micronuclei were found to contain material almost exclusively from $\mathrm{Chr} 1$, and rarely from Chr 16 (Figure 1c). Micronuclei observed in the ICF2 cell line contained Chr 1 and/or Chr 16 material with an equivalent frequency (Figure 1d). Chr 9 was rarely involved in any type of abnormality.

Finally, using FISH with specific probes, we demonstrated that micronuclei only contained the long arm of chromosome 1 and/or 16 with the juxtacentromeric heterochromatin, in the three ICF cell lines (data not shown). This result is in agreement with the fact that alpha satellite centromeric sequences are not generally involved in the chromosomal abnormalities observed in ICF syndrome, as they are essentially AT-rich.

In conclusion, cytogenetic abnormalities observed in all three ICF cell lines are characteristic of the syndrome and, moreover, they allow the ICF2 cell line to be clearly distinguished from the ICF1 and ICF3 cell lines.

\section{Aberrant nuclear distribution of HP1 $\alpha, \mathrm{HP} 1 \beta$ and HP1 $\gamma$ proteins in ICF patients}

As ICF syndrome is characterized by abnormalities of the heterochromatin, we searched for alterations in the distribution of one of its essential components, the HP1 protein. Using immunocytochemistry, we examined the in vivo subcellular distribution of $\mathrm{HP} 1 \alpha, \mathrm{HP} 1 \beta$, and $\mathrm{HP} 1 \gamma$, proteins in PHA-stimulated lymphocytes and lymphoblastoid cells derived from either ICF or normal subjects.

In both cell types from normal subjects, the most frequently observed HP1 $\alpha$ pattern consisted of nuclei showing bright irregularly shaped areas co-localizing with intense DAPI-stained heterochromatic regions, against a pale diffuse nucleoplasmic signal. This predominant pattern probably represents $\mathrm{G} 1$ nuclei, which is consistent with the duration of this phase of the cell cycle and previous observations $^{21,22}$ (Figure $2 \mathrm{a}, \mathrm{G} 1$ ). A less common HP1 $\alpha$ nuclear pattern consisted of discrete small speckles and a more distinct diffuse staining of the nucleoplasm. The DAPI-stained chromatin of the corresponding nuclei indicated that they were at the G2 phase $^{22}$ (Figure $2 \mathrm{a}, \mathrm{G} 2$ ). The HP1 $\beta$ and HP1 $\gamma$ patterns observed in control subjects appeared very similar to those of HP1 $\alpha$. Nevertheless, the diffuse staining of the nucleoplasm obtained with HP1 $\gamma$ protein was stronger than that obtained with $\mathrm{HP} 1 \beta$, which was in turn stronger than that obtained with $\mathrm{HP} 1 \alpha$, as described previously. ${ }^{21,23}$

In both cell types from ICF patients, the most frequently observed HP1 $\alpha$ pattern was identical to the one observed in G1 nuclei from normal patients. In addition, there was a striking pattern, consisting of nuclei showing one (or occasionally two) large bright $\mathrm{HP} 1 \alpha$ focus, with discrete speckles in the nucleoplasm that resembled the pattern from normal G2 nuclei (Figure 2b). By screening 200 nuclei 

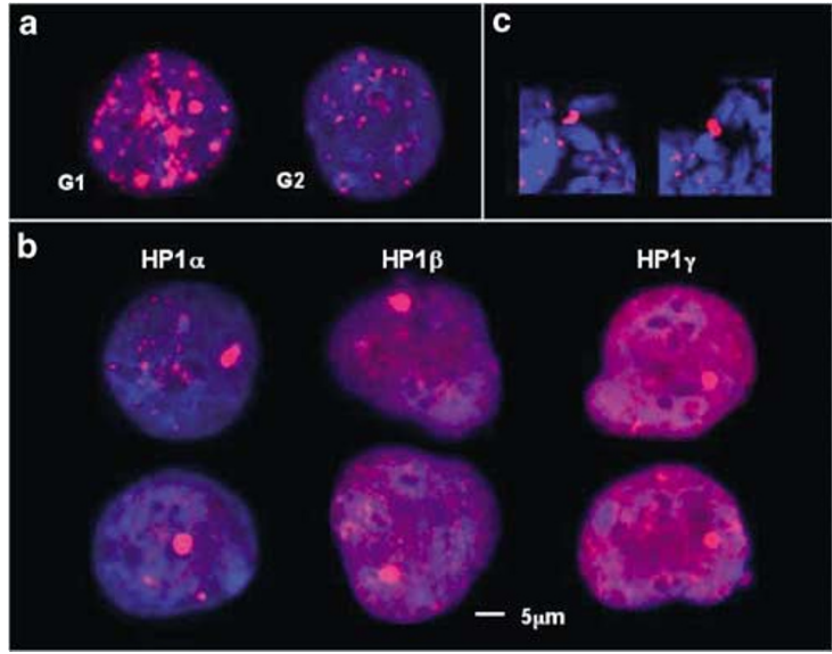

Figure 2 Nuclear distribution of HP1 proteins after immunostaining of normal and ICF cells: (a) HP1 $\alpha$ distribution in normal G1 and G2 nuclei. (b) In ICF cells, all three HP1 isoforms show a large intense HP1 focus, over a variably diffuse staining of the nucleoplasm. The diffuse staining of the nucleoplasm obtained with HP1 $\gamma$ was stronger than that obtained with $\mathrm{HP} 1 \beta$, which was in turn stronger than that obtained with $\mathrm{HP} 1 \alpha$ (scale bar: $5 \mu \mathrm{m})$. (c) Two partial prophases showing a large HP1 $\alpha$ focus onto the undercondensed $1 \mathrm{qh}$ heterochromatin.

from each lymphoblastoid cell line, the percentage of nuclei showing this aberrant pattern was evaluated to be around $25 \%$ in ICF patients, and less than $7 \%$ in the control subjects. In nuclei with the aberrant pattern, the intensity of the unique $\mathrm{HP} 1 \alpha$ focus appeared to be very strong, possibly reflecting a high concentration of protein in this nuclear domain. In addition, this intense focus was evaluated to be between 2 and $4 \mu \mathrm{m}$ in diameter. Immunocytochemistry with anti-HP1 $\beta$ and anti-HP1 $\gamma$ antibodies showed patterns similar to those observed with anti-HP1 $\alpha$ antibody, particularly the prominent bright focus pattern, which, as expected, was associated with a more intense staining of the nucleoplasm (Figure 2b).

We also examined the in vivo subcellular distribution of $\mathrm{HP} 1 \alpha, \mathrm{HP} 1 \beta$ and HP1 $\gamma$ proteins in fibroblasts of patient ICF1, but did not detect any abnormal pattern in this cell type.

The prominent bright HP1 focus co-localizes with 1qh and/or 16qh heterochromatin

The chromosome abnormalities observed in ICF patients predominantly affect $1 \mathrm{qh}$ and $16 \mathrm{qh}$ heterochromatin regions, and to a lesser extent those of $9 \mathrm{qh}$. Therefore, we investigated the relationship between these heterochromatin regions and the aberrant HP1 patterns observed in the nuclei of ICF patients.

For each ICF patient, 100 nuclei showing an intense HP1 $\alpha$ focus were photographed and subsequently hybri- dized with the specific 1qh heterochromatin probe and photographed again. We observed a high frequency of colocalization between the intense $\mathrm{HP} 1 \alpha$ focus and at least one 1qh heterochromatin, in 94/100 ICF1 nuclei, 82/100 ICF2 nuclei and 95/100 ICF3 nuclei (Figure 3). Subsequent hybridization to the same nuclei with the Chr 16 centromere probe gave at least one signal that co-localized with the bright HP1 $\alpha$ focus in 23/100 ICF1 nuclei, 53/100 ICF2 nuclei and 29/100 ICF3 nuclei (Figure 3). Finally, the ICF nuclei were hybridized with the $9 \mathrm{qh}$ heterochromatin probe, which was rarely found to be associated with the intense HP1 $\alpha$ focus: 5/100 ICF1 nuclei, and 4/100 ICF2 and ICF3 nuclei.

The frequencies with which the bright HP1 $\alpha$ focus colocalizes with 1qh (pale grey circles), 16qh (dotted circles) and $9 \mathrm{qh}$ (dark grey circles) satellite DNA probes are shown in Figure 4, for the three ICF patients. Interestingly, these frequencies appear to reflect the relative rate of involvement of each chromosome in cytogenetic abnormalities of the three patients. Cells from the ICF1 patient were immunostained with HP1 $\beta$ and HP1 $\gamma$ antibodies, and specific $1 \mathrm{qh}$ and $16 \mathrm{qh}$ probes hybridized secondarily on the same nuclei. HP1 $\beta$ and HP1 $\gamma$ bright foci co-localized with each heterochromatin probe, in proportions similar

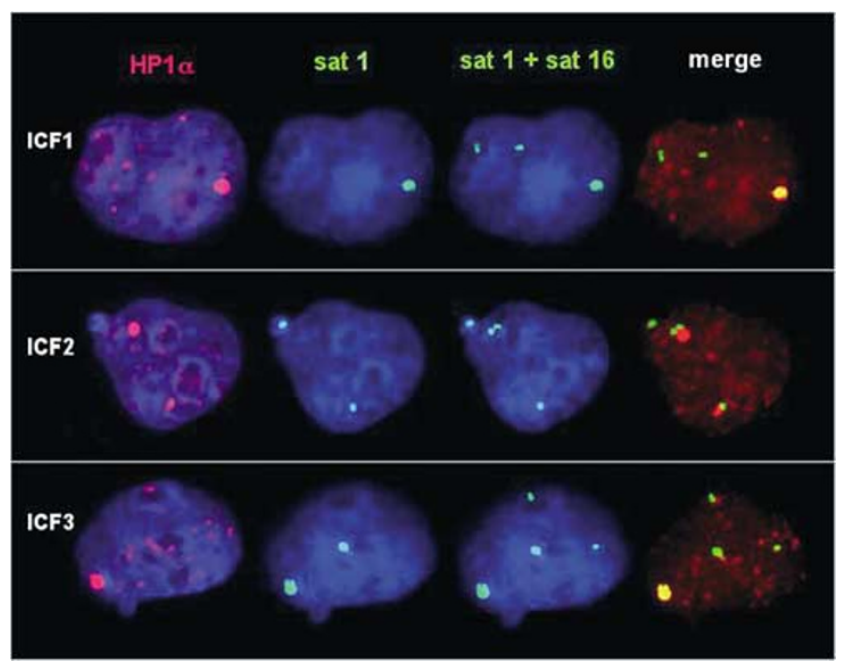

Figure 3 Representative ICF nuclei showing the colocalization of the intense HP1 $\alpha$ focus with satellite DNAs: Each horizontal line represents the same nucleus, first immunostained with anti-HP1 $\alpha$ antibody, then hybridized with $1 \mathrm{qh}$ satellite probe (green) and finally with $16 \mathrm{qh}$ satellite probe (green). All signals are merged in the last image. In the ICF1 nucleus, the heterochromatins of both chromosomes 1 (1 $\mathrm{qh})$, but not that of chromosomes 16 (16qh), co-localize with the bright HP1 $\alpha$ focus. In the ICF2 nucleus, the heterochromatins of both chromosomes 16 (16qh), but not that of chromosomes 1 (1qh), co-localize with the bright $\mathrm{HP} 1 \alpha$ focus. In the ICF3 nucleus, the heterochromatin of only one chromosome 1 co-localizes with the bright $\mathrm{HP} 1 \alpha$ focus. 

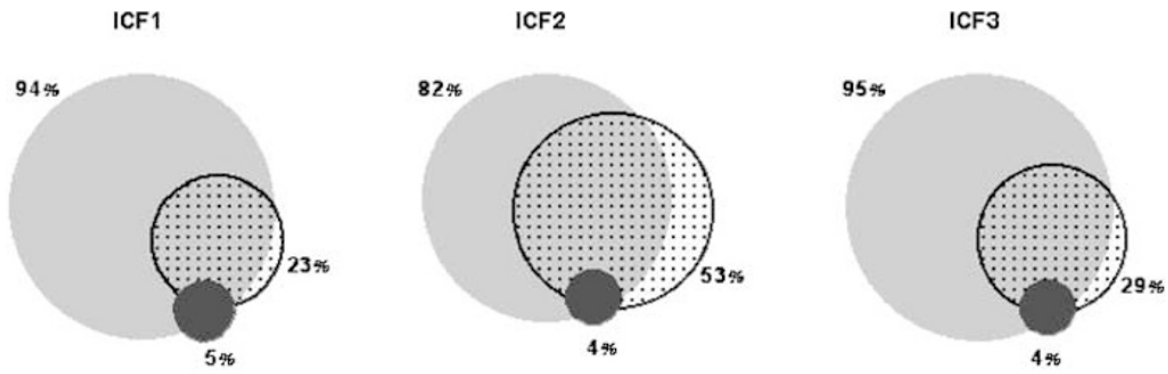

Co-localization of HP $1 \boldsymbol{\alpha}$. focus with heterochromatin :

1 qh

Figure 4 Schematic representation of the co-localization between HP1 $\alpha$ bright focus and satellite DNAs in ICF cell lines: For each cell line, 100 nuclei with a bright HP1 $\alpha$ focus were selected and hybridized with satellite DNA probes. Pale gray circles represent the percentages of co-localization between $\mathrm{HP} 1 \alpha$ and at least one $1 \mathrm{qh}$ satellite DNA. Dotted circles represent the percentages of co-localization between $\mathrm{HP} 1 \alpha$ and at least one 16qh satellite DNA. Dark gray circles represent the percentages of co-localization between $\mathrm{HP} 1 \alpha$ and at least one $9 \mathrm{qh}$ satellite DNA.

to that observed with HP1 $\alpha$, demonstrating that all HP1 isoforms have the same behaviour in ICF cells.

\section{The aberrant HP 1 pattern occurs at the G2 phase of} the cell cycle

To further characterize the time at which protein HP1 $\alpha$ concentrates in a prominent bright focus, the lymphoblastoid cells from ICF patients and controls were synchronized at different phases of the cell cycle.

In cells synchronized at late G1 phase, nuclei with a bright HP1 $\alpha$ focus were poorly represented in all subjects: $<15 \%$ in ICF patients and $<7 \%$ in controls (Figure 5 ). Cells blocked at the mid-S phase showed an increase in the percentage of nuclei with the aberrant HP1 $\alpha$ pattern, particularly in ICF patients. Cells from ICF patients collected at the G2 phase showed the highest percentages of nuclei with a bright HP1 $\alpha$ focus. Indeed, these percentages reached $62.3,54.8$ and $42.3 \%$ for ICF1, ICF2 and ICF3 patients, respectively. Cells from normal subjects, treated with the same G2-phase protocol, showed a clearly lower percentage of nuclei with aberrant HP1 $\alpha$ pattern $(\mathrm{C} 1$ : 14.3\%, C2: $14.5 \%$ and C3: 11.6\%). Finally, in cells synchronized at the metaphase stage (M-phase), the percentages of nuclei with a prominent bright HP1 $\alpha$ focus decreased in all cell lines, returning to the levels observed at the $S$ phase. Interestingly, we observed that, in rare prophases showing a bright HP $1 \alpha$ focus, this co-localized with one undercondensed 1qh heterochromatin, suggesting that such an HP1 pattern may reflect abnormalities in the condensation of this heterochromatin (Figure $2 \mathrm{c}$ ).

In conclusion, in all phases of the cell cycle, the percentage of nuclei showing the aberrant HP1 $\alpha$ pattern was significantly higher for ICF patients than for the controls (Figure 5). Moreover, it was clear that the percentage of nuclei with a bright HP1 $\alpha$ focus reached its greatest value at the G2 phase, for all the cell lines analyzed.

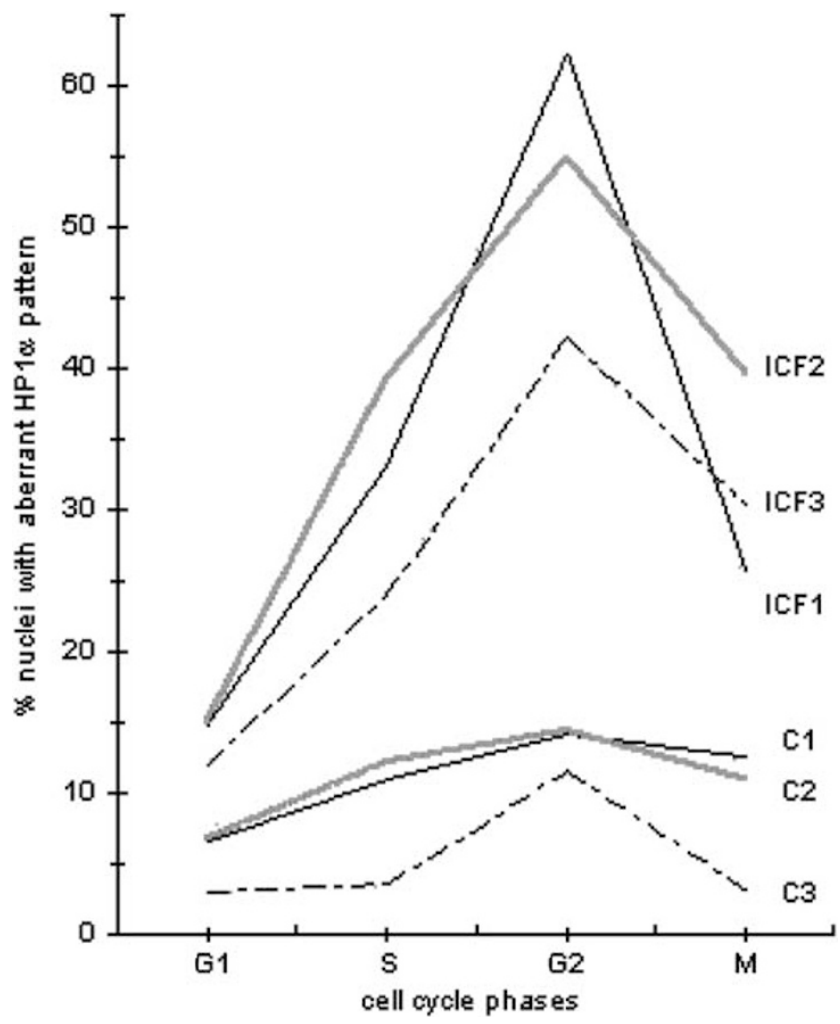

Figure 5 Distribution of nuclei with a bright HP1 $\alpha$ focus throughout the cell cycle: The ICF1, ICF2, ICF3 and control cell lines $(C 1,2,3)$ were treated in the same way in order to be synchronized at various phases of the cell cycle. The percentage of ICF nuclei with aberrant HP1 $\alpha$ pattern reached its highest value at the $G 2$ phase.

\section{Determining the partners of HP1 proteins that co- localize to the bright focus}

As HP1 proteins are known to interact with numerous proteins, we tested whether some of their known partners were present at the bright HP1 focus. 
First, we determined the subcellular distribution of SP100 that interacts directly with HP1 $\alpha$ protein. ${ }^{24}$ The G2 cells from ICF patients were immunostained with an antiSP100 antibody and showed that a high proportion of nuclei have a prominent bright SP100 focus, in addition to a diffuse faint staining of the nucleoplasm. Double immunocytochemistry with differently labelled HP $1 \alpha$ and SP100 antibodies clearly demonstrated their perfect nuclear co-localization to the bright focus (Figure 6a). The SP100 and PML proteins are associated in the NBs, and we therefore studied the nuclear distribution of PML protein in ICF cell lines synchronized in G2. We observed a high percentage of nuclei with a large bright PML focus. Each of these was secondarily demonstrated to associate with the 1qh heterochromatin (Figure 6c). The NB protein SUMO-1, which is known to modify covalently both SP100 and PML proteins, ${ }^{25}$ was also observed to give an aberrant bright focus co-localized with $\mathrm{HP} 1 \alpha$ (Figure $6 \mathrm{~b}$ ). The DAXX protein, also known to participate in the structure of PML NBs, was demonstrated to co-localize with prominent bright HP1 foci in ICF cell nuclei (data not shown).

Since HP1 protein isoforms are known to play a key role in the organization of heterochromatin, we next examined whether other hallmarks of the heterochromatic state are present at the bright HP1 focus. The linear anti-methylated histone H3-K9 Ab, which normally recognizes dimethyl H3-K9, showed the same diffuse signal in both normal and ICF nuclei; The branched $4 \times$ dimethyl H3-K9 Ab, which has a strong reactivity with trimethylated $\mathrm{H} 3-\mathrm{K} 9{ }^{26}$ appeared to be concentrated at DAPI-stained heterochro-

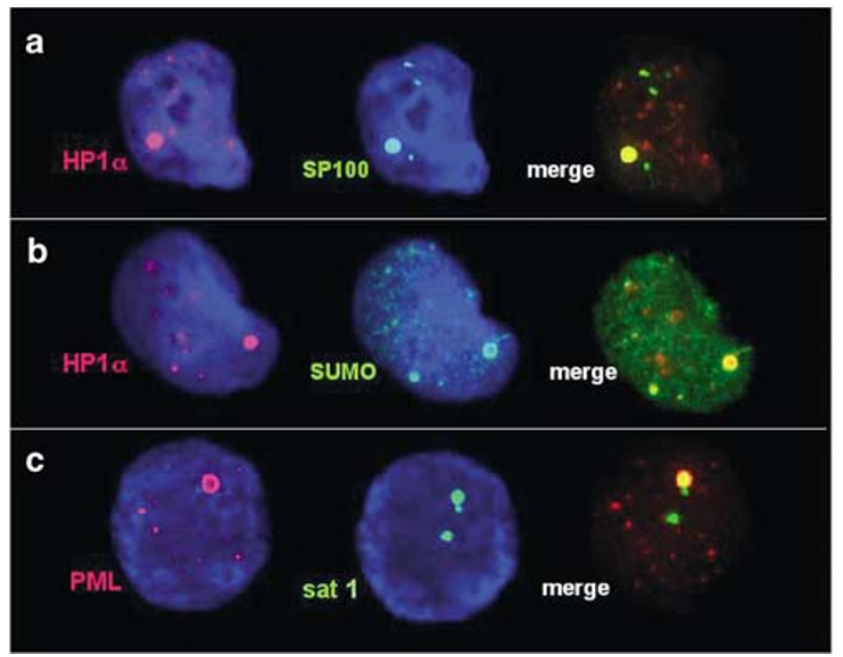

Figure 6 In ICF G2 nuclei, the bright HP1 $\alpha$ focus perfectly co-localizes with proteins from the PML NBs: (a) the same nucleus immunostained with anti-HP1 $\alpha \mathrm{Ab}$, then antiSP100 Ab and merged; (b) the same nucleus immunostained with anti-HP1 $\alpha$ Ab, then anti-SUMO-1 and merged; (c) the same nucleus immunostained with anti-PML Ab, then hybridized with $1 \mathrm{qh}$ satellite DNA probe. matic foci in both normal and ICF nuclei at the G1 phase (Figure 7a). As expected, in normal G2 nuclei, these foci appeared to be replaced by a more diffuse signal. Nevertheless, in ICF G2 nuclei, no strong signal was observed at the bright HP1 focus (Figure 7a). The anti-SUVAR39H1, the anti-DNMT3B, the anti-RB and the anti-DNMT1 $15,27,28,29$ Abs were also tested and showed no specific signal colocalizing with the bright HP1 focus, in ICF nuclei. Similarly, antibodies directed against members of the NuRD complex: histone de-acetylase HDAC1, Mi-2 and IKAROS, ${ }^{30}$ were tested, as antibody against MECP2, a methyl CpG-binding protein, ${ }^{31}$ and against the Histone H1 which characterizes condensed chromatin. ${ }^{32}$ Once again, no specific concentration of these proteins was observed to co-localize with the bright HP1 focus in ICF nuclei.

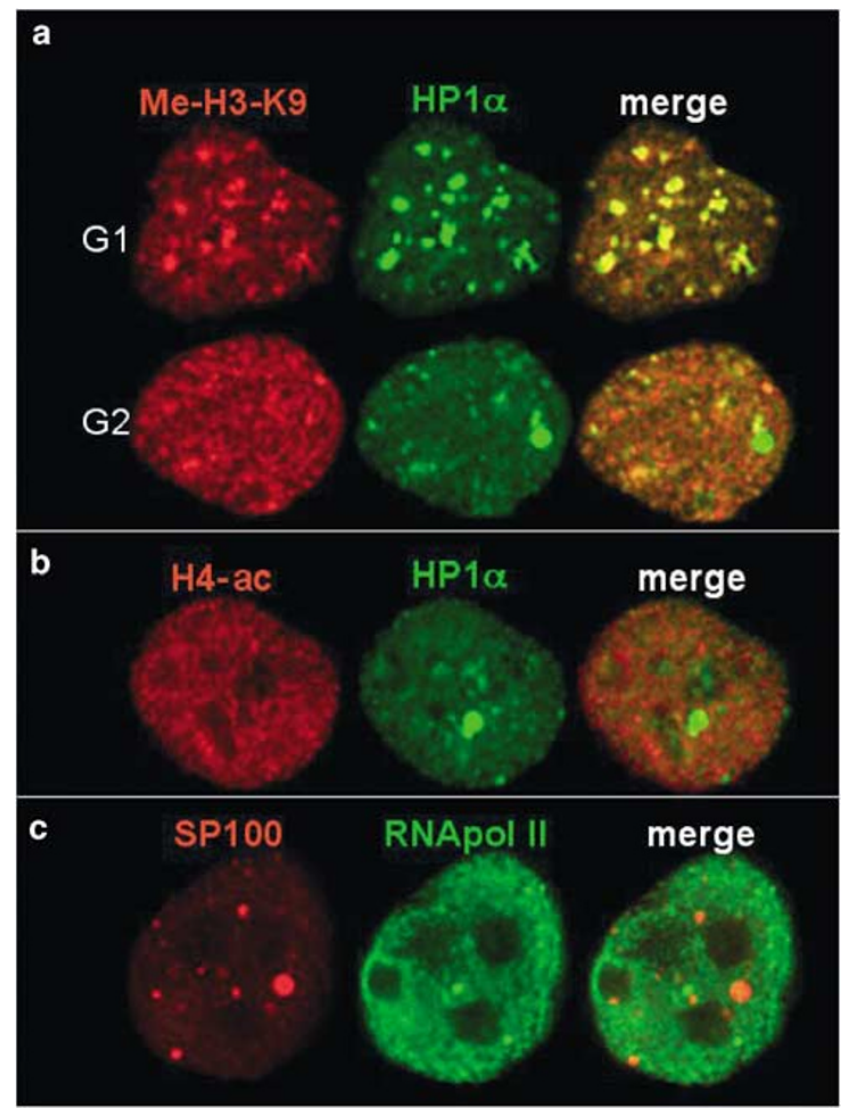

Figure 7 In ICF nuclei, the bright HP1 focus neither colocalizes with the usual hallmarks of heterochromatin, nor with those of the active chromatin: (a) the same G1 nucleus and the same G2 nucleus immunostained with anti-4 $\times$ methyl H3-K9 Ab (red), then anti-HP1 $\alpha$ Ab (green) and merged; (b) the same $G 2$ nuclei immunostained with anti-polyacetylated-H4 Ab (red), then $\mathrm{HP} 1 \alpha \mathrm{Ab}$ (green) and merged; (c) the same G2-nuclei immunostained with antiSP100 Ab (red), then anti RNA-pol II Ab (green), and merged. 
Since the known partners of HP1 in heterochromatin were absent from the bright HP1 focus, and HP1 and PML proteins have been shown to associate with transcriptionally active chromatin, ${ }^{33-35}$ we searched for the presence of hallmarks of active chromatin at the bright focus. The distribution of the histone $\mathrm{H} 3$ acetylated on K9 and K18 (data not shown), the poly-acetylated histone $\mathrm{H} 4$ (Figure $7 \mathrm{~b}$ ), and the phosphorylated RNA polymerase II (Figure 7c) was examined, but none of them showed any specific signal co-localizing with the bright protein focus. These results suggest that the bright HP1 focus observed in ICF G2 nuclei does not constitute an active chromatin domain.

\section{Discussion \\ Satellite DNA hypomethylation does not prevent HP1 protein localization to heterochromatin}

Classical satellite DNA is normally heavily methylated at cytosine residues, but in ICF syndrome patients it is almost completely unmethylated in all tissues. ${ }^{5}$ The cytogenetic abnormalities which characterize this syndrome suggest that satellite DNA hypomethylation may induce alterations in the structure and stability of heterochromatin ${ }^{6}$ and possibly in the nuclear distribution of one of its essential components, the HP1 proteins. Nevertheless, we show that the in vivo distribution of HP1 protein isoforms is only perturbed in G2 nuclei from ICF lymphoblastoid cell lines, where we observed HP1 to be concentrated in a prominent bright focus.

We demonstrated that the bright HP1 focus is closely associated with decondensed $1 \mathrm{qh}$ and/or $16 \mathrm{qh}$ heterochromatin. This result, in addition to the normal HP1 distribution observed at other phases of the cell cycle, demonstrates that satellite DNA hypomethylation does not prevent the HP1 proteins from localizing to the heterochromatin. A similar result has been obtained in Dnmt3a/ Dnmt3b double-deficient mouse ES cells, since in this model impaired DNA methylation does not alter HP1 $\alpha$ localization at pericentric heterochromatin. ${ }^{29}$ These observations are in agreement with the fact that HP1 binding to heterochromatin results from de-acetylation and methylation of histone tails, both of which are epigenetic imprints more stable, and better conserved throughout evolution, than the DNA methylation imprint. ${ }^{36}$

Our results also demonstrate that, in ICF G2 nuclei, HP1 proteins concentrate in a prominent focus whose intensity may reflect a high concentration of protein. It is now known that the target for HP1 binding is trimethylated H3K9. ${ }^{29}$ Nevertheless, it is improbable that the intense HP1 focus results from an increase in methylated $\mathrm{H} 3-\mathrm{K} 9$, as the branched $4 \times$ dimethyl H3-K9 antibody did not show an intense fluorescent signal at this site. This result suggests that HP1 proteins concentrate at the bright focus through another mode of targeting.
Interestingly, HP1 proteins have been suggested to act in different ways in the formation of heterochromatin, since they retain their ability to bind to histones $\mathrm{H} 3$ even when their N-terminal domain has been removed. ${ }^{10}$ In addition, it has been shown that HP1 $\alpha$ may associate with pericentic heterochromatin depending not only on its methylbinding chromo domain but also on an RNA-binding activity present in the hinge region of the protein. ${ }^{37,38}$ The lack of acetylated histones $\mathrm{H} 3$ and $\mathrm{H} 4$ at the bright HP1 focus, as well as the lack of phosphorylated RNA Pol II, demonstrates that the HP1 bright focus is not an active chromatin domain, but cannot exclude the presence of RNA at this site.

\section{The bright HP1 focus may reflect incomplete condensation of hypomethylated heterochromatin at G2 phase}

The percentage of nuclei with a bright HP1 focus was the highest in ICF cells synchronized at the G2 phase. This phase of the cell cycle is characterized by chromosome condensation that normally initiates from the centromeric region, then spreads in cis throughout each chromosome arm and is completed before mitosis. The Drosophila HP1 protein has been shown to be essential for proper chromosome condensation in vivo, ${ }^{39}$ and its high degree of amino-acid identity with human isoforms suggests that they all have similar functions. ${ }^{40}$

In ICF G2 nuclei, we observed a reduction of HP1 signal, except on the $1 \mathrm{qh}$ and/or $16 \mathrm{qh}$ heterochromatin, where an intense focus of HP1 $\alpha$ persisted, specifically associated with undercondensed heterochromatin. This is in agreement with the pattern observed during the cell cycle in normal cells, where only a small portion of $\mathrm{HP} 1 \alpha$ is retained on centromeric heterochromatin between G2 and prophase. ${ }^{22}$ We may suppose that, in normal G2/M nuclei, HP1 proteins are associated with heterochromatin until its condensation is completed. In ICF patients, heterochromatin may find it difficult to condense rapidly at the G2 phase when its satellite DNA is hypomethylated and, consequently, HP1 proteins could remain accumulated at these sites during a longer period. The G2/condensation phase has been demonstrated to be longer in fibroblasts than in lymphocytes ${ }^{41}$ or lymphoblastoid cells (personal observations). This may explain the lack of either chromosomal abnormalities or the aberrant HP1 pattern in ICF fibroblasts, despite satellite DNA hypomethylation.

Interestingly, the DNMT3B methyltransferase has recently been shown to co-immunoprecipitate with multiple components of the condensin complex, as well as the remodeling enzyme hSNF2. ${ }^{42}$ Therefore, mutations in DNMT3B may alter its ability to interact with these components and thus reduce the efficiency of heterochromatic condensation, in ICF patients.

Finally, since incomplete condensation of 1qh and 16qh often leads to unresolved Holliday junctions, it could be 
the primary cause of all other chromosomal abnormalities observed in the ICF syndrome, including micronuclei. ${ }^{6}$

\section{In ICF nuclei, the bright HP1 focus co-localizes with a giant PML nuclear body}

The SP100 protein, a major component of the PML NBs, and the SUMO-1 protein were shown to co-localize to the bright HP1 focus.

In normal mammalian cells, the SP100 protein is known to directly interact with all three members of the HP1 family through their chromo shadow domain. ${ }^{24}$ In addition, SP100 can be covalently modified by the ubiquitinlike SUMO-1 protein. Since the SUMO-1 and ubiquitin proteins target the same lysines, it has been suggested that sumoylation of proteins antagonizes their ubiquitination, and thus protects them from ubiquitin-mediated degradation. ${ }^{43}$ The stabilization of protein complexes by SUMO modification, as shown with SUMO-SP100 and HP1, ${ }^{25}$ has been proposed to have a similar effect on the mobility and localization of the SUMO target protein or its partner(s). Our results suggest that, despite the reduced nuclear localization of the majority of HP1 protein at the G2 phase, some HP1 protein might be concentrated and stabilized by complexing with SP100-SUMO-1 on the 1qh and/or 16qh heterochromatin.

We show that the PML protein co-localizes with SP100 and the HP1 bright focus observed in ICF nuclei. The PML protein is critical for NB formation and is responsible for the proper localization of all other NB-associated proteins. ${ }^{44}$ PML needs to be covalently modified by SUMO-1 in order to localize to the NBs, and only sumoylated PML acquires the capacity to bind and to recruit DAXX to the NBs. ${ }^{44}$ Thus, the simultaneous presence of PML, SUMO-1 and DAXX, in addition to SP100 and HP1 isoforms in bright HP1 foci, indicates that these structures are NBs.

The large size of the bright PML focus in ICF G2 nuclei, $2-4 \mu \mathrm{m}$ in diameter, leads us to propose that it is a giant NB. Normal NBs contain a number of proteins ${ }^{16}$ and their biological functions have not been well defined. It was initially suggested that they were involved in multiple cellular activities, including transcriptional regulation, growth regulation and antiviral defense. Moreover, because many proteins with different functions aggregate in NBs, it has been suggested that these bodies function as nuclear depots. ${ }^{44}$ The in vivo associations between PML and several DNA repair proteins, including BLM, Mre11, Rad51 and $\mathrm{H} 2 \mathrm{AX}$, support a role for the $\mathrm{NB}$ in the maintenance of genome integrity and in DNA repair. ${ }^{45,46}$ These DNA repair functions are highly relevant to the ICF syndrome. Indeed, cytogenetic abnormalities observed in this syndrome not only involve undercondensed heterochromatin regions but also chromosome breaks and multibranched configurations.

\section{Heterochromatin polymorphism may underlie ICF phenotypic variability}

It is not clear how hypomethylation of satellite DNA leads to the broad variation in phenotypic characteristics seen in ICF syndrome patients. It is also reasonable to speculate that the lack of DNA methyltransferase activity leads to derepression of silenced genes, but the target genes for DNMT3B have not yet been identified. Furthermore, the paucity of ICF patients, coupled with the molecular heterogeneity of the disease, ${ }^{4}$ implies that it is not possible to establish genotype-phenotype correlations.

Our study suggests a factor that may underlie ICF phenotypic variability: polymorphism in heterochromatin length. Heterochromatic regions of the human genome are generally considered to be genetically inert, and consequently their large variability in size (length polymorphism) is thought to have no effect on phenotype. Nevertheless, we find that the abnormal bright HP1 protein focus associates with uncondensed 1qh and 16qh heterochromatins with different frequencies in different ICF patients, reflecting the extent to which these chromosomes are involved in cytogenetic abnormalities and micronuclei. Our results suggest that the frequency with which a chromosome is involved in abnormalities is directly related to the time taken to condense its heterochromatin during G2.

We hypothesize that the longer a 1qh or 16qh heterochromatin is, the less quickly it will condense when its satellite DNA is hypomethylated, and the more frequently it will be involved in cytogenetic abnormalities. In support of this, the ICF1 patient has higher percentage of nuclei with a bright HP1 focus, higher percentage of chromosome abnormalities and more severe clinical phenotype than the ICF2 patient. In addition, such a length polymorphism might explain why chromosome 1 , which is rich in satellite 2 DNA as is chromosome 16, but generally has longer heterochromatin, is more frequently involved in cytogenetic abnormalities in the ICF syndrome. It might also explain why both ICF patients from the same sibship present very different phenotypes. ${ }^{47}$. Therefore, for an ICF patient with a given genotype, the size of the 1qh and 16qh heterochromatins, which corresponds to the amount of satellite $\mathrm{DNA}^{48}$ could influence the severity of the phenotype.

In conclusion, we have studied ICF syndrome using the original approach of characterizing the distribution of HP1 proteins in vivo. All HP1 protein isoforms appear to be clustered in one bright HP1 focus in ICF cells synchronized at G2 phase. Each intense HP1 focus perfectly co-localizes with 1qh and/or 16qh undercondensed heterochromatin, suggesting that satellite DNA hypomethylation does not prevent HP1 protein localizing to heterochromatin. Moreover, proteins specifically involved in the formation of PML NBs also co-localize with the intense HP1 focus, forming a giant PML body. Such giant NBs might 
constitute an interesting model for understanding the multiple functions of these nuclear structures.

\section{Acknowledgements}

We thank Isabelle Thuret (Service d'Hématologie Pédiatrique, Hôpital d'enfants de la Timone, Marseille) for referring the patients, the families for their cooperation and Valérie Delague for her help in everything. We would like to thank Pierre Chambon \& Cécile Egly (IGBMC, Illkirch, France), Thomas Jenuwein \& Antoine Peters (IMP, Vienna, Austria), Amanda Fischer (MRC, London, UK), Marc Vigneron and Claude Kedinger (ESBS, Strasbourg, France), Claire Vourc'h and Caroline Jolly (IAB, Grenoble, France), Sharon $R$ Dent (DBMB, Houston, Texas, USA), Vincent Géli (CNRS, Marseille, France), and Françoise Birg (Inserm U119, Marseille, France) for kindly providing antibodies. We also thank Mike Mitchell for helpful discussion and revision of the text. This work was supported by grants from the ARC (Association pour la Recherche contre le Cancer), INSERM, the Ministère de l'Enseignement et de la Recherche. Judith Luciani is supported by a grant from the 'Fondation Electricite de France' (EDF).

\section{References}

1 Maraschio P, Zuffardi O, Dalla Fior T, Tiepolo L: Immunodeficiency, centromeric heterochromatin instability of chromosomes 1, 9, and 16, and facial anomalies: the ICF syndrome. J Med Genet 1988; 25: 173-180.

2 Hansen RS, Wijmenga C, Luo $\mathrm{P}$ et al: The DNMT3B DNA methyltransferase gene is mutated in the ICF immunodeficiency syndrome. Proc Natl Acad Sci USA 1999; 96: $14412-14417$

$3 \mathrm{Xu}$ GL, Bestor TH, Bourc'his D et al: Chromosome instability and immunodeficiency syndrome caused by mutations in a DNA methyltransferase gene. Nature 1999; 402: 187-191.

4 Wijmenga C, Hansen RS, Gimelli G et al: Genetic variation in ICF syndrome: evidence for genetic heterogeneity. Hum Mutat 2000; 16: $157-509$.

5 Jeanpierre M, Turleau C, Aurias A et al: An embryonic-like methylation pattern of classical satellite DNA is observed in ICF syndrome. Hum Mol Genet 1993; 2: 731-735.

6 Tuck-Muller CM, Narayan A, Tsien F et al: DNA hypomethylation and unusual chromosome instability in cell lines from ICF syndrome patients. Cytogenet Cell Genet 2000; 89: 121-128.

7 James TC, Elgin SC: Identification of a nonhistone chromosomal protein associated with heterochromatin in Drosophila melanogaster and its gene. Mol Cell Biol 1986; 6: 3862-3872.

8 Eissenberg JC, James TC, Foster-Hartnett DM, Hartnett T, Ngan V, Elgin SC: Mutation in a heterochromatin-specific chromosomal protein is associated with suppression of position-effect variegation in Drosophila melanogaster. Proc Natl Acad Sci USA 1990; 87: 9923-9927.

9 Festenstein R, Sharghi-Namini S, Fox M et al: Heterochromatin protein 1 modifies mammalian PEV in a dose- and chromosomalcontext-dependent manner. Nat Genet 1999; 23: 457-461.

10 Nielsen AL, Oulad-Abdelghani M, Ortiz JA, Remboutsika E, Chambon P, Losson R: Heterochromatin formation in mammalian cells: interaction between histones and HP1 proteins. Mol Cell 2001; 7: 729-739.

11 Cheutin T, McNairn AJ, Jenuwein T, Gilbert DM, Singh PB, Misteli $\mathrm{T}$ : Maintenance of stable heterochromatin domains by dynamic HP1 binding. Science 2003; 299: 721-725.

12 Jenuwein T, Allis CD: Translating the histone code. Science 2001; 293: $1074-1080$.

13 Grewal SI, Elgin SC: Heterochromatin: new possibilities for the inheritance of structure. Curr Opin Genet Dev 2002; 12: 178-187.
14 Bannister AJ, Zegerman P, Partridge JF et al: Selective recognition of methylated lysine 9 on histone H3 by the HP1 chromo domain. Nature 2001; 410: 120-124.

15 Lachner M, O'Carroll D, Rea S, Mechtler K, Jenuwein $\mathrm{T}$ : Methylation of histone $\mathrm{H} 3$ lysine 9 creates a binding site for HP1 proteins. Nature 2001; 410: 116-120.

16 Seeler JS, Dejean A: The PML nuclear bodies: actors or extras? Curr Opin Genet Dev 1999; 9: 362-367.

17 Carpenter NJ, Filipovich A, Blaese RM, Carey TL, Berkel AI: Variable immunodeficiency with abnormal condensation of the heterochromatin of chromosomes 1, 9, and 16. J Pediatr 1988; 112: $757-760$.

18 Spadari S, Pedrali-Noy G, Falaschi MC, Ciarrocchi G: Control of DNA replication and cell proliferation in eukaryotes by aphidicolin. Toxicol Pathol 1984; 12: 143-148.

19 Webber LM, Garson OM: Fluorodeoxyuridine synchronization of bone marrow cultures. Cancer Genet Cytogenet 1983; 8: 123-132.

20 Mignon-Ravix C, Depetris D, Delobel B, Croquette MF, Mattei MG: A human interstitial telomere associates in vivo with specific TRF2 and TIN2 proteins. Eur J Hum Genet 2002; 10: 107-112.

21 Minc E, Allory Y, Worman HJ, Courvalin JC, Buendia B: Localization and phosphorylation of HP1 proteins during the cell cycle in mammalian cells. Chromosoma 1999; 108: 220-234.

22 Sugimoto K, Tasaka H, Dotsu M: Molecular behavior in living mitotic cells of human centromere heterochromatin protein HPLalpha ectopically expressed as a fusion to red fluorescent protein. Cell Struct Funct 2001; 26: 705-718.

23 Metzler-Guillemain C, Luciani J, Depetris D, Guichaoua MR, Mattei MG: HP1beta and HP1gamma, but not HP1alpha, decorate the entire $\mathrm{XY}$ body during human male meiosis. Chromosome Res 2003; 11: 73-81.

24 Seeler JS, Marchio A, Sitterlin D, Transy C, Dejean A: Interaction of SP100 with HP1 proteins: a link between the promyelocytic leukemia-associated nuclear bodies and the chromatin compartment. Proc Natl Acad Sci USA 1998; 95: 7316-7321.

25 Seeler JS, Dejean A: Nuclear and unclear functions of SUMO. Nat Rev Mol Cell Biol 2003; 4: 690-699.

26 Peters AH, O'Carroll D, Scherthan $\mathrm{H}$ et al: Loss of the Suv39h histone methyltransferases impairs mammalian heterochromatin and genome stability. Cell 2001; 107: 323-337.

27 Geiman TM, Sankpal UT, Robertson AK et al: Isolation and characterization of a novel DNA methyltransferase complex linking DNMT3B with components of the mitotic chromosome condensation machinery. Nucleic Acids Res 2004; 32: 2716-2729.

28 Nielsen SJ, Schneider R, Bauer UM et al: Rb targets histone H3 methylation and HP1 to promoters. Nature 2001; 412: 561-565.

29 Lehnertz B, Ueda Y, Derijck AA et al: Suv39h-mediated histone H3 lysine methylation directs DNA methylation to major satellite repeats at pericentric heterochromatin. Curr Biol 2003; 13: $1192-1200$

30 Ahringer J: NuRD and SIN3 histone deacetylase complexes in development. Trends Genet 2000; 16: 351-356.

31 Lewis JD, Meehan RR, Henzel WJ et al: Purification, sequence, and cellular localization of a novel chromosomal protein that binds to methylated DNA. Cell 1992; 69: 194-905.

32 Shen X, Yu L, Weir JW, Gorovsky MA: Linker histones are not essential and affect chromatin condensation in vivo. Cell 1995; 82: $47-56$.

33 Piacentini L, Fanti L, Berloco M, Perrini B, Pimpinelli S: Heterochromatin protein 1 (HP1) is associated with induced gene expression in Drosophila euchromatin. J Cell Biol 2003; 161: 707-714.

34 Wang J, Shiels C, Sasieni P et al: Promyelocytic leukemia nuclear bodies associate with transcriptionally active genomic regions. $J$ Cell Biol 2004; 164: 515-526.

35 Ehrlich M, Buchanan KL, Tsien F et al: DNA methyltransferase 3B mutations linked to the ICF syndrome cause dysregulation of lymphogenesis genes. Hum Mol Genet 2001; 10: 2917-2931.

36 Wakefield MJ, Keohane AM, Turner BM, Graves JA: Histone underacetylation is an ancient component of mammalian 
X chromosome inactivation. Proc Natl Acad Sci USA 1997; 94: 9665-9668.

37 Muchardt C, Guilleme M, Seeler JS, Trouche D, Dejean A, Yaniv $\mathrm{M}$ : Coordinated methyl and RNA binding is required for heterochromatin localization of mammalian HP1alpha. EMBO Rep 2002; 3: 975-981.

38 Maison C, Bailly D, Peters AH et al: Higher-order structure in pericentricheterochromatin involves a distinct pattern of histone modification and an RNA component. Nat Genet 2002; 30: 329_ 334.

39 Kellum R, Alberts BM: Heterochromatin protein 1 is required for correct chromosome segregation in Drosophila embryos. J Cell Sci 1995; 108: 1419-1431.

40 Singh PB, Georgatos SD: HP1: facts, open questions, and speculation. J Struct Biol 2002; 140: 10-16.

41 Maraschio P, Tupler R, Dainotti E, Piantanida M, Cazzola G, Tiepolo L: Differential expression of the ICF (immunodeficiency, centromeric heterochromatin, facial anomalies) mutation in lymphocytes and fibroblasts. J Med Genet 1989; 26: 452-456.

42 Geiman TM, Sankpal UT, Robertson AK et al: Isolation and characterization of a novel DNA methyltransferase complex linking DNMT3B with components of the mitotic chromosome condensation machinery. Nucleic Acids Res 2004; 32: 2716-2729.

43 Desterro JM, Rodriguez MS, Hay RT: SUMO-1 modification of IkappaBalpha inhibits NF-kappaB activation. Mol Cell 1998; 2: 233-239.

44 Ishov AM, Sotnikov AG, Negorev D et al: PML is critical for ND10 formation and recruits the PML-interacting protein daxx to this nuclear structure when modified by SUMO-1. J Cell Biol 1999; 147: $221-234$.

45 Zhong S, Hu P, Ye TZ, Stan R, Ellis NA, Pandolfi PP: A role for PML and the nuclear body in genomic stability. Oncogene 1999; 18: $7941-7947$.

46 Carbone R, Pearson M, Minucci S, Pelicci PG: PML NBs associate with the hMre11 complex and p53 at sites of irradiation induced DNA damage. Oncogene 2002; 21: 1633-1640.

47 Gimelli G, Varone P, Pezzolo A, Lerone M, Pistoia V: ICF syndrome with variable expression in sibs. J Med Genet 1993; 30: $429-432$.

48 Gosden JR, Lawrie SS, Cooke HJ: A cloned repeated DNA sequence in human chromosome heteromorphisms. Cytogenet Cell Genet 1981; 29: 32-39. 